LAWRENCE LIVERMORE N A T IO N A L LABORATORY

Floating into Thin Air

A. U. Hazi

February 8, 2007 
This document was prepared as an account of work sponsored by an agency of the United States Government. Neither the United States Government nor the University of California nor any of their employees, makes any warranty, express or implied, or assumes any legal liability or responsibility for the accuracy, completeness, or usefulness of any information, apparatus, product, or process disclosed, or represents that its use would not infringe privately owned rights. Reference herein to any specific commercial product, process, or service by trade name, trademark, manufacturer, or otherwise, does not necessarily constitute or imply its endorsement, recommendation, or favoring by the United States Government or the University of California. The views and opinions of authors expressed herein do not necessarily state or reflect those of the United States Government or the University of California, and shall not be used for advertising or product endorsement purposes.

This work was performed under the auspices of the U.S. Department of Energy by University of California, Lawrence Livermore National Laboratory under Contract W-7405-Eng-48. 


\section{Floating into Thin Air}

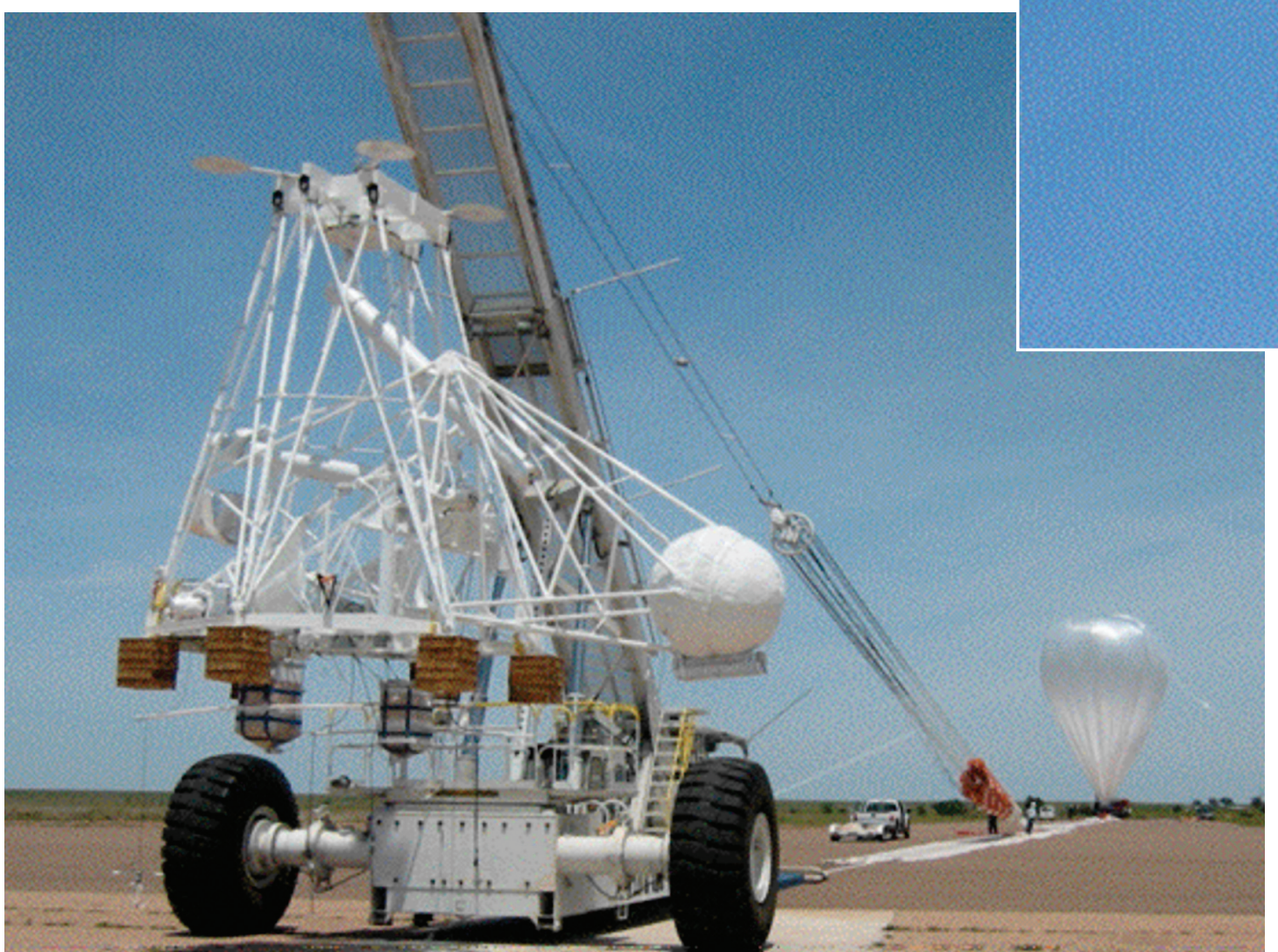

Two wire cables connect the gondola of the High Energy Focusing Telescope (HEFT) to its balloon. By the time the balloon reaches its float altitude (inset) 40 kilometers above Earth's surface, it has expanded enough to fill the space of a football stadium.

N May 18, 2005, a giant helium balloon carrying the High Energy Focusing Telescope (HEFT) sailed into the spring sky over the deserts of New Mexico. The spindly steel and aluminum gondola that houses the optics, detectors, and other components of the telescope floated for 25 hours after its launch from Fort Sumner, New Mexico. For 21 of those hours, the balloon was nearly 40 kilometers above Earth's surface - almost four times higher than the altitude routinely flown by commercial jet aircraft. In the upper reaches of Earth's atmosphere, HEFT searched the universe for x-ray sources from highly energetic objects such as binary stars, galaxy clusters, and supermassive black holes.

Before landing in Arizona, the telescope observed and imaged a dozen scientific targets by capturing photons emitted from these objects in the high-energy (hard) $\mathrm{x}$-ray range (above
10 kiloelectronvolts). Among these targets were the $\mathrm{Crab}$ synchrotron nebula, the black hole Cygnus X-1 (one of the brightest $\mathrm{X}$-ray sources in the sky), and the blazar 3C454.3. The scientific data gathered from these targets are among the first focused hard x-ray images returned from high altitudes.

"It went up, it worked, and it collected data," says Livermore physicist Bill Craig, who is part of the multi-institution collaboration that built and launched the telescope. HEFT is actually an array of co-aligned telescopes that together provide the unique high-sensitivity (sub-arcminute) resolution and fewarcsecond positioning required to achieve its science goals. Each telescope consists of a grazing incidence optics module that focuses onto a shielded solid-state pixel detector. (See $S \& T R$, November 2004, pp. 4-11.) In designing HEFT, the collaboration, 
which includes researchers from Lawrence Livermore, the California Institute of Technology, Columbia University, and the Danish Space Research Institute, capitalized on the special core competencies of each institution. With Laboratory Directed Research and Development funding, the Livermore team engineered HEFT's optics and designed and built the gondola.

Craig is clearly pleased with the success of the flight. "We collected far more information from hard x-ray sources than with our earlier generation of instruments," he says. "A lot of people will be working on the data for years to come."

\section{$X$ Marks the Spot}

$\mathrm{X}$-ray telescopy is not new. Coverage of the low-energy (soft) $\mathrm{x}$-ray band (up to 12 kiloelectronvolts) - by missions involving the National Aeronautics and Space Administration's (NASA's) Chandra X-Ray Observatory and the multinational XMM satellite - has been excellent and thorough.

HEFT takes the basic principles of these soft $x$-ray missions and moves into the 10- to 70-kiloelectronvolt range, where the demands for accuracy and precision in engineering the telescope's optics are much higher. Because of its range and relatively large effective collecting area of about 60 square centimeters, HEFT is well suited for imaging objects such as supernova remnants and galaxy clusters. HEFT is also ideal for detecting objects obscured by galactic dust.

Active galactic nuclei (AGN) —-some of the brightest extragalactic X-ray sources - are distant galaxies with massive black holes at their centers. AGN contribute significantly to the $\mathrm{x}$-ray background of the universe, which is of great interest to astrophysicists in studying the origins of the universe. Although
AGN have been observed in the soft x-ray band, many of them are obscured by dust clouds that soft $\mathrm{x}$ rays cannot penetrate. However, the high-energy particles of these dust-covered AGN can be detected with the hard x-ray focusing apparatus of HEFT.

Retrieving large amounts of data from the HEFT mission was not the only great success according to Craig. Despite the expected vagaries of balloon missions, the launch itself went off without a hitch. "Balloon launches are completely unpredictable," says Craig. "We never know what's going to happen."

The balloon, which is made from a material similar to a plastic bag, swells to the size of a football stadium by the time it reaches its float altitude at 40 kilometers. At the time of launch, the helium occupies only the very top of the balloon, giving the balloon a teardrop shape. Once an experiment is completed, explosives are triggered, causing the balloon to rip open. As the gondola drops to Earth, a parachute deploys to aid in the landing. A flight crew follows the landing balloon to recover the gondola and equipment.

\section{Starlight, Star Bright}

HEFT spent about 20 hours of its mission focusing on targets. Its detectors needed only a few minutes to confirm the presence of bright sources such as Cygnus X-1 and the Crab. Fainter sources required several hours to confirm detection. HEFT and NASA's Swift x-ray telescope simultaneously observed the extragalactic blazar 3C454.3. Because the blazar was under eruption during the HEFT flight, it was one of the brightest AGN in the sky.

"What we look for in the data is spectral variation," says Craig. "We've got a lot of data to analyze now." The first analysis task, currently under way, is aspect reconstruction to determine the pointing position of the telescope versus time.

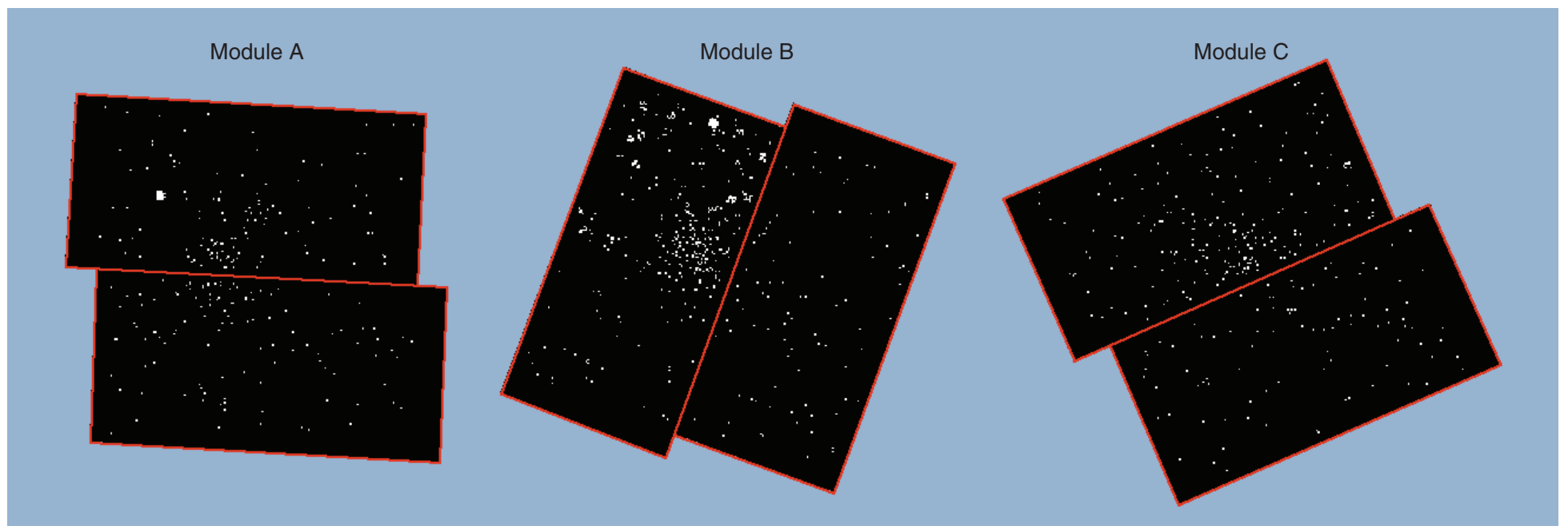

HEFT's three high-energy x-ray focusing optics modules recorded these first light images (now being analyzed) of the Crab Nebula. Because this target was extremely bright, the high-energy telescope needed only 5 minutes to record the data. 


\section{Out of This World}

Craig is eager to keep HEFT afloat. A mission is scheduled for 2007 in Australia, where HEFT's detectors and star trackers will be pointed at the center of the galaxy in the southern sky. Also slated is a launch from Sweden in 2008. On that flight, the balloon will cross the Arctic Circle to Canada at a time when Cassiopeia A will be in HEFT's field of view for five days. Cassiopeia A is a supernova remnant that is thought to be 327 years old.

Plans are already under way to take HEFT to the next level. NASA's Small Explorer Program has approved the Nuclear Spectroscopic Telescope Array (NuSTAR), a follow-on project to HEFT. NuSTAR takes HEFT's x-ray focusing abilities and sends them beyond Earth's atmosphere on a satellite. The optics design and the proposed production process for NuSTAR are based on those used to build the HEFT telescopes. "NuSTAR is opening one of the few remaining frontiers in space," says Craig. "With it, we will be able to bring the high-energy universe into focus."

NuSTAR will be hundreds of times more sensitive than any previous hard $\mathrm{x}$-ray instrument, which will greatly improve image resolution. It will orbit Earth at an altitude of about
480 kilometers for 3 years, during which time researchers intend to take a census of black holes. They hope to measure both the rate at which black holes are growing and the accretion rate at which material has fallen into black holes over time.

Craig underscores the importance of Livermore's contribution to the two missions. "Both NuSTAR and HEFT fit perfectly with Livermore's core competencies in hard x-ray and high-energy astrophysics research," he says. "It's important that we keep flying the HEFT missions - so we can answer some primary research questions and demonstrate what can be done."

- Maurina S. Sherman

Key Words: active galactic nuclei (AGN), balloon launch, black holes, hard x rays, High Energy Focusing Telescope (HEFT), Nuclear Spectroscopic Telescope Array (NuSTAR), soft x rays.

For further information contact Bill Craig (925) 423-1471 (billc@IInl.gov).

\section{HEFT and NuSTAR Update}

Researchers are continuing to analyze the data from the May 2005 HEFT balloon flight and preparing to publish intriguing scientific results on several of the observed astronomical sources. The HEFT balloon gondola and detectors are being rebuilt in preparation for a flight from Alice Springs, Australia in 2008. Launching from that site allows the HEFT telescope to observe a number of sources in the center of our galaxy that cannot be observed from the Northern Hemisphere. The Alice Springs launch site also offers the possibility of a very long flight, 2 days or more aloft, which would allow the observation of a broad range of x-ray emitting objects. An entirely new type of detector that could offer significantly more sensitivity to certain phenomena will be tested during the 2008 flight.

The NuSTAR program successfully completed the first phase of development, but NASA canceled the program in 2006 for budgetary reasons as the agency realigns to support its new priorities. The NuSTAR concept is still under development and may be submitted in response to future NASA solicitations.

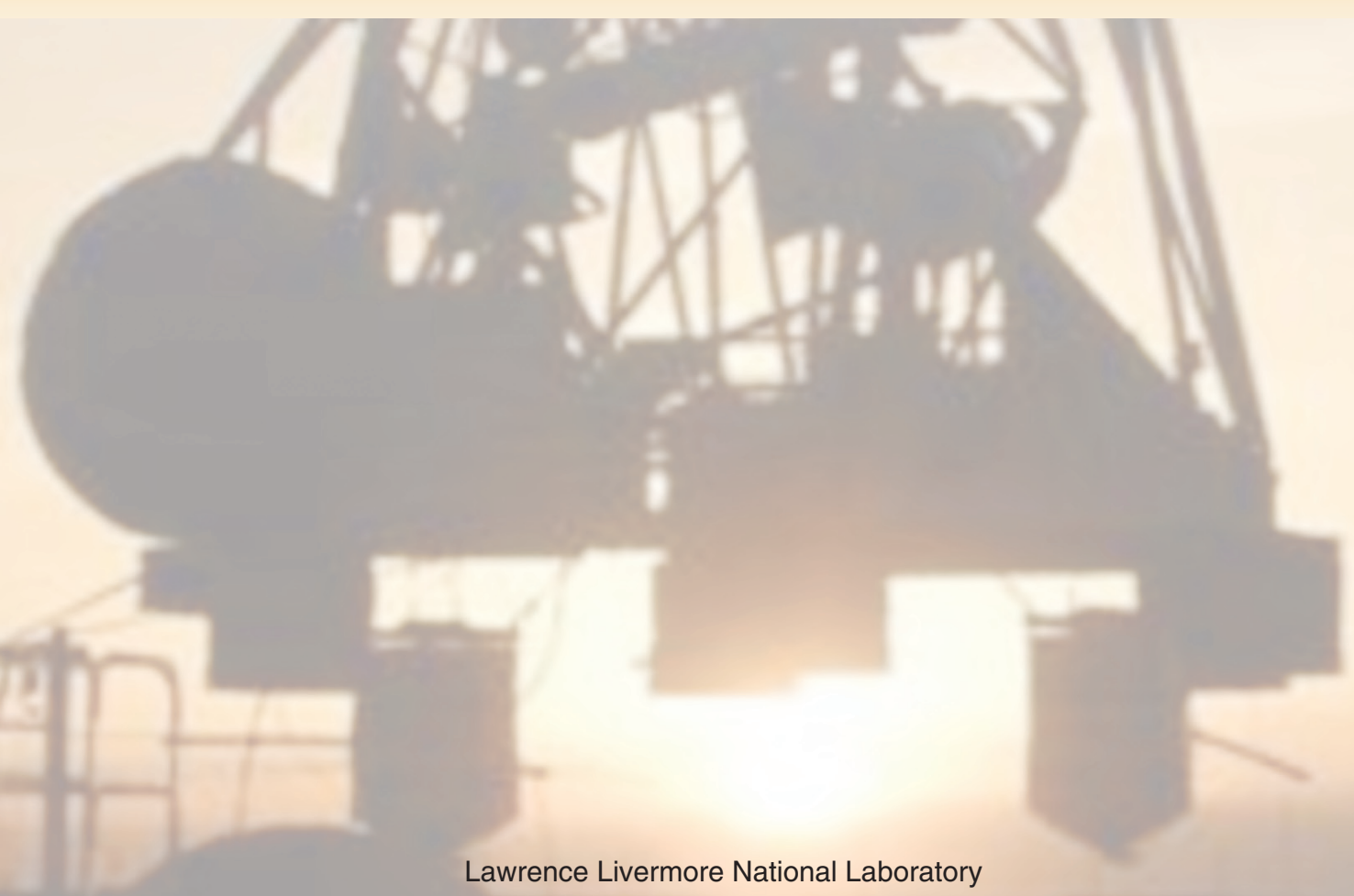




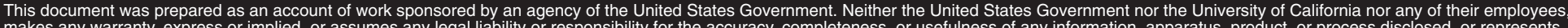

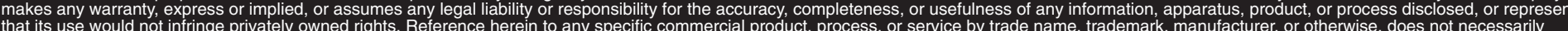

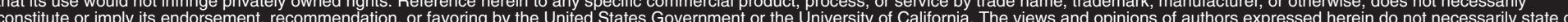
reflect those of the United States Government or the University of California, and shall not be used for advertising or product endorsement purposes.

This work was performed under the auspices of the U.S. Department of Energy by University of California, Lawrence Livermore National Laboratory under Contract W-7405-Eng-48. 